\title{
Practice of Interventional Radiology during the COVID-19 Pandemic
}

\author{
Shyamkumar N. Keshava ${ }^{1}$ Arun Gupta ${ }^{2}$ Rochan Pant ${ }^{3}$ Patrick D. Sutphin ${ }^{4}$ Sanjeeva Kalva ${ }^{4}$ \\ ${ }^{1}$ Department of Interventional Radiology, Division of Clinical \\ Radiology, Christian Medical College, Tamil Nadu, India \\ 2Department of Interventional Radiology, Sir Ganga Ram Hospital, \\ New Delhi, India \\ ${ }^{3}$ Department of Radiology, Sir HN Reliance Foundation Hospital, \\ Mumbai, Maharashtra, India \\ ${ }^{4}$ Division of Interventional Radiology, Department of Radiology, \\ Massachusetts General Hospital, Boston, Massachusetts, \\ United States
}

J Clin Interv Radiol ISVIR 2020;4:1-2

The coronavirus disease 2019 (COVID-19) pandemic is expanding rapidly across the globe, with more cases being detected daily. ${ }^{1}$ The rapid expansion of the disease is attributed to both droplet transmission of the virus ${ }^{2}$ and globalization of human travel. A severe shortage of testing kits, lack of facilities for widespread testing, and low sensitivity of the testing methods make it difficult to know the true burden of the pandemic.

The limited information from China, Europe, and the United States (the most severely affected regions in the world) has been guiding our leaders on managing the pandemic in India. As per the data, nearly $80 \%$ of the affected individuals are asymptomatic but can potentially spread the virus if not isolated. Among the symptomatic $(\sim 20 \%)$, the disease appears to be very severe, requiring ventilatory support in 3 to $4 \%$ and lethal in 1 to $3 \%$. The limited resources (especially the intensive care unit beds, ventilators, etc.) and lack of scalability of existing hospitals to manage the severely symptomatic patients have directed the leaders to focus on limiting the rapid spread of the pandemic, thereby "flattening the curve," so that the number of symptomatic people will be well within the threshold capacity of the resources. Given that there is not yet any herd immunity and no effective cure or vaccine, preventative measures are the only method to decrease the spread of the pandemic. As droplet transmission occurs through infected respiratory droplets $(>5-10 \mu \mathrm{m})$ in a close range, typically within $1 \mathrm{~m}$, one of the most effective methods to stem disease transmission is physical distancing. The current distance recommendation in the United States for physical distancing, also known as "social distancing," is
6 feet $(\sim 2 \mathrm{~m})$. In addition, this includes avoiding congregate settings and mass gatherings. Several regions and countries have called for lockdowns to enforce physical isolation. As droplet transmission also occurs through fomites such as doorknobs and stethoscopes, adjunct methods to reduce the risk of transmission include meticulous personal hygiene (washing hands with soap and water) and avoiding touching the face with hands. Medical personnel can limit exposure to the virus by using personal protection equipment (PPE) while working with patients suspected of or affected by COVID-19.

As interventional radiology (IR) practitioners, we are experiencing COVID-19-related challenges every day. Elective interventional procedures have been restricted as part of lockdown and reorganizing the resources and preserving precious PPE within the hospitals. Appropriate screening of the patients is required prior to an emergency IR procedure so that the medical personnel involved can consider appropriate precautions. The Indian government has declared a 3-week lockdown, ${ }^{3}$ during which the medical personnel have been advised to work in such a way that only a proportion of the manpower is in the hospital on a rotating basis. Based on this, most hospitals have advised the staff to work for 1 week and stay at home for 2 weeks during the lockdown period. If there is a necessity to perform an IR procedure on a positive or suspected COVID-19 patient, one must follow safe precautions (wearing PPE and N95 masks for aerosol-generating procedures). One needs to be up to date regarding PPE as the guidelines may be modified based on new information. Due to the shortage of resources, medical personnel are advised to reuse surgical face masks, face shields, and N95 masks.

\footnotetext{
Address for correspondence Shyamkumar N. Keshava, DMRD, DNB, FRCR, FRANZCR, Department of Interventional Radiology, Division of Clinical Radiology, Christian Medical College, Ida Scudder Road, Vellore 632004, Tamil Nadu, India (e-mail: shyamkumar.n.keshava@ gmail.com).
}

DOI https://doi.org/ 10.1055/s-0040-1709962 ISSN 2457-0214. C2020 by Indian Society of Vascular
and Interventional Radiology

Published online

April 14, 2020

\section{License terms}

() (1) $\Theta \circledast$ 
The medical personnel from IR (nurses and physicians) may be redeployed across the hospital to take care of COVID-19 patients.

The IR societies have swiftly responded. COVID-19 clinical notification from the Society of Interventional Radiology (SIR) provides guidelines for IR practice. ${ }^{4}$ These include cancelling all procedures except those that are urgent or emergent, minimizing the use of essential items, screening all patients for high-risk exposure or symptoms according to the Centers for Disease Control and Prevention guidelines, and identifying the aerosol-generating IR procedures and optimal use of PPE, optimizing staffing and constituting teams to facilitate social distancing, and limiting risk of community spread. ${ }^{3}$ The Cardiovascular Interventional Society of Europe (CIRSE) has provided information to the members in the following categories: general information, preparedness based on past pandemics, and preparedness with respect to checklists, diagnosis, and treatment approaches. ${ }^{5} \mathrm{~A}$ webinar has been conducted by the Asia Pacific Society of Cardiovascular Interventional Radiology (APSCVIR) to sensitize the IR practitioners regarding safe practice of IR during this pandemic. Many IR societies, including the APSCVIR, SIR, and ISVIR, have cancelled the scheduled annual conferences.

As members of the public, we have our responsibility to follow the rule of the land. This includes all the steps to contain the spread of the virus, especially by social distancing and hygiene. There is overwhelming information regarding COVID-19 in social media. We could perhaps play a role in identifying the genuine relevant information and passing it to our friends and families.

We hope collectively we will be able to face the global challenge of the COVID-19 pandemic. We also hope that we will be able to recover from the personal and financial losses from this pandemic. Hopefully, we will gain more knowledge and wisdom on dealing with such pandemics in future.

Conflict of Interest

None declared.

\section{References}

1 World health Organization. Coronavirus disease (COVID-19) Pandemic. Available at: https://www.who.int/emergencies/ diseases/novel-coronavirus-2019. Accessed March 31, 2020

2 World health Organization. Modes of transmission of virus causing COVID-19: implications for IPC precaution recommendations. Available at: https://www.who.int/news-room/commentaries/ detail/modes-of-transmission-of-virus-causing-covid-19-implications-for-ipc-precaution-recommendations. Accessed March 31, 2020

3 New York Times. Modi Orders 3-Week Total Lockdown for All 1.3 Billion Indians. Available at: www.nytimes.com/2020/03/24/ world/asia/india-coronavirus-lockdown.html. Accessed March 31, 2020

4 Society of Interventional Radiology. COVID-19 resources for IR. Available at: www.sirweb.org/practice-resources/ COVID-19-resources. Accessed March 31, 2020

5 Cardiovascular Interventional Society of Europe. OVID-19 resource centre. Available at: www.cirse.org/education/COVID19-resource-centre. Accessed March 31, 2020 